\title{
The Veronesean of quadrics and associated loci
}

By J. W. Head, Canford School, Wimborne, Dorset.

(Received 31 st October, 1935; received in revised form 1st January, 1936. Read 1st November, 1935.)

In this paper we consider the correspondence between tangential quadrics of [3] and points of [9]. Godeaux ${ }^{1}$ has considered this geometrically, with the object of obtaining a representation for a twisted cubic of three dimensions. We have considered it from a standpoint more algebraic than that of Godeaux, with particular reference to the types of pencils of quadrics that correspond to special lines of [9], and to the interpretation in [9] of the fact that the condition for a net of quadrics to be part of the polar system of a cubic surface is poristic.

$\S 1$. We denote a general point of [3] by $P_{x}$, its coordinates being $\left(x_{0}, x_{1}, x_{2}, x_{3}\right)$ and its equation $P_{x}=0$.

We denote a general plane of [3] by $\Pi_{X}$, its coordinates being $\left(X_{0}, X_{1}, X_{2}, X_{3}\right)$ and its equation $\Pi_{X}=0$.

The equation of a locus quadric $q$ is $\Sigma a_{i j} x_{i} x_{j}=0$. The equation of a tangential quadric $Q$ is $\Sigma A_{i j} X_{i} X_{j}=0$, and $q$ is therefore apolar to $Q$ if $\Sigma a_{i j} A_{i j}=0$.

The suffixes $i, j$ are always interchangeable and have the values $0,1,2,3$.

We denote by $Q_{y}$ a general point of [9] whose coordinates are $\left(y_{00}, y_{11}, y_{22}, y_{33} ; y_{12}, y_{20}, y_{01} ; y_{03}, y_{13}, y_{23}\right)$. The point $Q_{A}$ or $\left(A_{00}, A_{11}\right.$, $\left.A_{22}, A_{33} ; A_{12}, A_{20}, A_{01} ; A_{03}, A_{13}, A_{23}\right)$ represents the quadric $Q$, while the prime $\tau_{a}$ or $\Sigma a_{i j} y_{i j}=0$ represents the quadric $q$. Hence if $\varpi_{a}$ contains $Q_{A}, q$ and $Q$ are apolar and conversely.

(3) 14 (1927).

1 "La géométrie de la cubique gauche," Bulletin de la Soc. Roy. des Sciences de Liége 
Consider the locus

$$
\left|y_{i j}\right|,=0,
$$

where the suffix $r$ indicates that the determinant $\left|y_{i j}\right|$ is of rank $r$.

If $r=3, Q_{y}$ represents a conic. The locus of such points is a variety $V_{8}^{4}$ of dimension 8 and order 4 . If $r=2, Q_{y}$ represents a point pair. The locus of such points is a variety $V_{\theta}^{10}$ of order 10 and dimension 6. If $r=1, Q_{y}$ represents a repeated point. The locus of such points is a variety $V_{3}^{8}$ of order 8 and dimension 3 whose parametric equations may be written

$$
y_{i j}=x_{i} x_{j}
$$

$V_{3}^{8}$ is therefore triple on $V_{8}^{4}$ and quadruple on $V_{6}^{10}$, while $V_{6}^{10}$ is double on $V_{8}^{4}$. We shall denote $(1,0,0,0)$ by $P_{0},(0,1,0,0)$ by $P_{1}$, $(0,0,1,0)$ by $P_{2}$ and $(0,0,0,1)$ by $P_{3}$. We shall denote by $Q_{x y}$ the point representing the point pair $P_{x} P_{y}=0$, whether $x=y$ or not.

§2. Representation of $V_{3}^{8}$ on a solid. The representation of $V_{3}^{8}$ on the solid is analogous to that of the Veronese surface on the plane, and we give a brief summary of corresponding elements and properties :-

\section{On Solid}

A point $P_{x}$

A curve of degree $n$

A surface of degree $n$

A plane

A quadric

Two quadrics have in general a unique common self-polar tetrad.

A quadric $\lambda P_{x}^{2}+\mu P_{y}^{2}=0$ is a point pair.

A quadric $\lambda P_{x}^{2}+\mu P_{y}^{2}+\nu P_{z}^{2}=0$ is a tangential conic.

\section{On $V_{3}^{8}$}

A point $Q_{x x}$

A curve of degree $2 n$

A surface of degree $4 n$

A Veronese surface

An octic del Pezzo surface ${ }^{\text {. }}$

There is a unique solid through a general line of [9] meeting $V_{3}^{8}$ in 4 points. Such a solid we call four-secant.

Chords of $V_{3}^{8}$ lie on $\nabla_{6}^{30}$.

Trisecant planes of $V_{3}^{8}$ lie on $V_{8}^{4}$.

Godeaux (loc. cit. \$4) obtains these results geometrically.

1 Represented by quartics with two fixed double points. 
§3. Flat spaces on $V_{8}^{4}$ and $V_{6}^{10}$. Tangent spaces.

Flat spaces on $V_{8}^{4}$ are of two kinds:-

(i) [5]'s $\sigma_{5}$ representing conics of a plane;

(ii) [4]'s $\sigma_{4}$ representing conics touching a line at a point.

There is one $\sigma_{5}$ through a general point of $V_{8}^{4}, \infty^{1}$ through a point of $V_{6}^{10}$ and $\infty^{2}$ through a point of $V_{3}^{8}$. The equations of a typical $\sigma_{5}$ are

$$
\text { - } \sum_{i=0}^{3} X_{i} y_{i j}=0, \quad(j=0,1,2,3) .
$$

Two spaces $\sigma_{5}$ representing conics in planes $\alpha, \beta$ meet in the plane of the conic representing the line $(\alpha \beta)$. The typical space $\sigma_{4}$ represents the system of conics

$$
\begin{aligned}
\left(z_{0} X_{0}+z_{1} X_{1}+z_{2} X_{2}+z_{3} X_{3}\right)\left(\lambda X_{0}\right. & \left.+\mu X_{1}+\nu X_{2}+\rho X_{3}\right) \\
& +\sigma\left(x_{0} X_{0}+x_{1} X_{1}+x_{2} X_{2}+x_{3} X_{3}\right)^{2}=0,
\end{aligned}
$$

and at any point of it the six expressions

$$
\left(2 z_{i} z_{j} y_{i j}-z_{i}^{2} y_{j j}-z_{j}^{2} y_{i i}\right) /\left(x_{i} z_{j}-x_{j} z_{i}\right)^{2}
$$

must be equal. There are $\infty^{5}$ spaces $\sigma_{4}$ altogether, $\infty^{1}$ through a general point of $V_{8}^{4}$ [Godeaux, loc. cit., § 7].

Flat spaces on $V_{6}^{10}$ are of two kinds:-

(i) Tangent solids $\sigma_{3}$ to $V_{3}^{8}$,

(ii) Planes $\sigma_{2}$ of conics on $V_{3}^{8}$ [Godeaux, loc. cit., §9].

Through a point $Q_{x y}$ on $V_{6}^{10}$ there is one plane $\sigma_{2}$, namely that of the conic on $V_{3}^{8}$ through $Q_{x x}$ and $Q_{y y}$. The $\infty^{1}$ spaces $\sigma_{4}$ through $Q_{x y}$ are obtained by joining it to the tangent solids $\sigma_{3}$ to $V_{3}^{8}$ at points of this conic.

The tangent prime at any point of $V_{8}^{4}$ meets $V_{3}^{8}$ in two Veronese surfaces, since it contains the space $\sigma_{5}$ through the point.

Consider next tangent [6]'s $\sigma_{6}$ to $V_{6}^{10}$. A space $\sigma_{6}$ represents tangential quadrics with a common generator $g$, and touches $V_{6}^{10}$ at all points of the plane $\gamma$ containing the conic of $V_{3}^{8}$ representing $g$. It meets $V_{8}^{4}$ in a repeated quadric plane cone of vertex $\gamma$. One system of generating [4]'s of this cone are spaces $\sigma_{4}$, while the other system 
consists of [4]'s each lying in a space $\sigma_{5}$. 'The equations of the tangent [6] at $Q_{x y}$, which joins the tangent solids to $V_{3}^{8}$ at $Q_{x x}$ and $Q_{y y}$ are:-

$$
\begin{aligned}
& 2 y_{02} B_{03} B_{32}+2 y_{23} B_{20} B_{03}+2 y_{30} B_{32} B_{20}+y_{00} B_{23}^{2}+y_{22} B_{30}^{2}+y_{33} B_{02}^{2}=0 \\
& 2 y_{01} B_{03} B_{31}+2 y_{13} B_{10} B_{03}+2 y_{30} B_{31} B_{10}+y_{00} B_{13}^{2}+y_{11} B_{30}^{2}+y_{33} B_{01}^{2}=0 \\
& 2 y_{01} B_{02} B_{21}+2 y_{12} B_{10} B_{02}+2 y_{20} B_{21} B_{10}+y_{00} B_{12}^{2}+y_{11} B_{20}^{2}+y_{22} B_{01}^{2}=0
\end{aligned}
$$

where $B_{i j}=x_{i} \xi_{j}-x_{j} \xi_{i}$ and therefore

$$
B_{01} B_{23}+B_{02} B_{31}+B_{03} B_{12}=0 .
$$

There are $\infty^{1}$ spaces $\sigma_{6}$ through a general point of $S_{9}$, and $\infty^{2}$ through a point of $V_{6}^{10}$.

A space $\sigma_{6}$ meets $V_{6}^{10}$ in a rational quartic plane cone, for the point pairs of an $\infty^{6}$ system of tangential quadrics with a common generator $g$ consist of a point of the generator and an arbitrary point, are therefore $\infty^{4}$, and can be put in $(1,1)$ correspondence with the points of a [4]. Any net of quadrics with a common generator has four point pairs, and hence follows the fact that the locus is quartic. The vertex of the cone is $\gamma$. It is possible by a process of elimination to prove that the only solids meeting $V_{6}^{10}$ in space curves are those which lie in spaces $\sigma_{6}$. In any space $\sigma_{6}$ there will be $\infty^{4}$ exceptional solids which are the intersection of two spaces $\sigma_{6}$ and meet $V_{6}^{10}$ in a quadric surface ( $c f$. Godeaux, loc. cit. $\$ 21$ ).

\section{§4. Lines of [9].}

Consider the various possible positions of a line in [9] with regard to $V_{8}^{4}$. A general line meets $V_{8}^{4}$ in 4 distinct points, but lines in special positions may meet $V_{6}^{10}$ or $V_{3}^{8}$ or touch or osculate $V_{8}^{4}$. We give below a table shewing the types of (tangential) quadrics corresponding to the various types of special position possible for a line of [9]. We thus obtain by varying the position of a line in [9] all the standard tangential quadrics dual to those given by Bromwich. ${ }^{1}$

1 Bromwich, Quadratic Forms and their classification by means of Invariant Factors, Cambridge Tract No. 3. 


\begin{tabular}{|c|c|c|}
\hline No. & $\begin{array}{l}\text { Segre characteristic and position } \\
\text { of line in [9]. }\end{array}$ & Base envelope of corresponding pencil in [3]. \\
\hline 1. & {$[1111]$; a general line. } & A general quartic developable of genus 1 . \\
\hline 2. & [(11) 11$]$; a line meeting $V_{0}{ }^{0}$. & $\begin{array}{l}\text { Two quadric cones with two common tangent } \\
\text { planes each touching all quadrics of the } \\
\text { pencil at a fixed point. }\end{array}$ \\
\hline 3. & $\begin{array}{l}{[(11)(11)] ; \text { a chord of } V_{6}^{\circ} \text { not }} \\
\text { on } V_{8}^{4} .\end{array}$ & $\begin{array}{l}\text { Four pencils of planes whose axes form a } \\
\text { skew quadrilateral. }\end{array}$ \\
\hline 4. & {$[(111) 1]$; a line meeting $V_{3}^{8}$. } & $\begin{array}{l}\text { A quadric cone twice; the quadrics of the } \\
\text { pencil have ring-contact. }\end{array}$ \\
\hline 5. & $\begin{array}{l}{[211] \text {; a line touching } V_{8}^{4} \text {, not }} \\
\text { meeting } V_{6}^{10} \text {. }\end{array}$ & $\begin{array}{l}\text { A quartic developable with a double tangent } \\
\text { plane, on which all quadrics of the pencil } \\
\text { have a common point. }\end{array}$ \\
\hline 6. & $\begin{array}{l}{[(21) 1] ; \text { a line touching } V_{8}^{4} \text { at }} \\
\text { a point of } V_{6}^{10} \text { (three-point con. } \\
\text { tact). }\end{array}$ & $\begin{array}{l}\text { generator } \\
\text { it. }\end{array}$ \\
\hline 7. & $\begin{array}{l}{[2(11)] ; \text { a tangent to } V_{8}^{4} \text { which }} \\
\text { meets } V_{6}^{10} \text {. }\end{array}$ & $\begin{array}{l}\text { A quadric cone and } \\
\text { whose axes meet and }\end{array}$ \\
\hline 8. & $\begin{array}{l}{[(211)] ; \text { a tangent to } V_{8}^{4} \text { at a }} \\
\text { point of } V \&(i . e . \text { having four- } \\
\text { point contact). }\end{array}$ & $\begin{array}{l}\text { Two pencils of planes through intersecting } \\
\text { axes, each counted twice. Quadrics of the } \\
\text { system have a common point on each axis. }\end{array}$ \\
\hline 9. & [22]; a bitangent to $V_{\frac{4}{4}}^{4}$. & $\begin{array}{l}\text { A pencil of planes through a line } l \text { and a } \\
\text { cubic developable, two of whose generating } \\
\text { planes contain } l \text {. }\end{array}$ \\
\hline 10. & {$[(22)] ;$ a tangent to $V_{t i}^{10}$} & $\begin{array}{l}\text { Three pencils of planes through skew lines } \\
l_{1}, l_{2} \text { and a transversal } l_{3} \text {, the last pencil } \\
\text { being counted twice. }\end{array}$ \\
\hline 11. & $\begin{array}{l}\text { [4]; a line having four-point } \\
\text { contact with } V_{8}^{4} \text {. }\end{array}$ & $\begin{array}{l}\text { A cubic developable and a pencil of planes } \\
\text { through a generator of it. }\end{array}$ \\
\hline 12. & {$[31] ;$ a line which osculates $V_{8}^{4}$. } & $\begin{array}{l}\text { A quartic developable with a stationary } \\
\text { generating plane. }\end{array}$ \\
\hline 13. & $\begin{array}{l}{[(31)] ; \text { a line through a point }} \\
\text { of } V_{\frac{1}{6}} \text { having four-point con- } \\
\text { tact with } V_{\frac{4}{8}}^{4} \text {. }\end{array}$ & $\begin{array}{l}\text { Two pencils of planes and a quadric cone } \\
\text { through the intersection of their axes, } \\
\text { touching the joining plane of those axes. }\end{array}$ \\
\hline 14. & $\begin{array}{l}\text { A line on } V_{8}^{4} \text { in a space } \sigma_{4} \\
\text { [No Segre Characteristic]. }\end{array}$ & $\begin{array}{l}\text { A pencil of planes counted twice and a } \\
\text { quadric cone. }\end{array}$ \\
\hline 15. & $\begin{array}{l}{[111] \text {; a line on } V_{6}^{4} \text { in a space }} \\
\sigma_{5} .\end{array}$ & $\begin{array}{l}\text { Four pencils of planes through four con- } \\
\text { current lines. }\end{array}$ \\
\hline
\end{tabular}




\section{§5. Planes of [9].}

The general plane meets $V_{8}^{4}$ in a non-singular quartic. Hesse in his paper on the bitangents of a non-singular plane quartic considered the condition for the general member of a net of (locus) quadrics to be a cone. If the net be $\lambda_{0} Q_{0}+\lambda_{1} Q_{1}+\lambda_{2} Q_{2}=0$, this condition is quartic in the $\lambda^{\prime}$ s. In the dual case we could take the $\lambda$-plane to be the plane representing the net and the quartic its intersection with $V_{8}^{4}$.

Any special relation of quadrics of a net must be reflected in a corresponding speciality of the quartic in which the representative plane meets $V_{8}^{4}$. This curve may be degenerate in various ways, including the extreme case of a line repeated four times, which occurs when the plane touches $V_{6}^{10}$ along a line or has 4-point contact with $V_{8}^{4}$ along a line. The latter occurs for the net

$$
2 a\left(X_{1} X_{2}-X_{0} X_{3}\right)+b X_{2}^{2}+2 c X_{1} X_{3}+2 d X_{2} X_{3}+e X_{3}^{2}=0
$$

(where $a, b, c, d$ are connected by two linear relations).

\section{§6. Solids of [9].}

In general a solid meets $V_{8}^{4}$ in a symmetroid. The most interesting special case is when this becomes a Kummer Surface. The Kummer Surface whose equation is in the standard form

where

$$
\left(\delta_{0} \lambda_{0} r_{0}\right)^{1 / 2}+\left(\delta_{1} \lambda_{1} r_{1}\right)^{1 / 2}+\left(\delta_{2} \lambda_{2} r_{2}\right)^{1 / 2}=0
$$

$$
\begin{aligned}
& \delta_{0}+\delta_{1}+\delta_{2}=0, \quad r_{0} \equiv a_{2} \lambda_{1}-a_{1} \lambda_{2}-b_{0} \lambda_{3}, \\
& r_{1} \equiv-a_{2} \lambda_{0}+a_{0} \lambda_{2}-b_{1} \lambda_{3}, \quad r_{2} \equiv a_{1} \lambda_{0}-a_{0} \lambda_{1}-b_{2} \lambda_{3},
\end{aligned}
$$

can be regarded as the condition for a quadric of the web

$$
\begin{gathered}
2 \lambda_{0}\left(\delta_{0} X_{1} X_{2}-a_{2} X_{1} X_{3}+a_{1} X_{2} X_{3}\right)+2 \lambda_{1}\left(\delta_{1} X_{0} X_{2}+a_{2} X_{0} X_{3}-a_{0} X_{2} X_{3}\right) \\
+2 \lambda_{2}\left(\delta_{2} X_{0} X_{1}-a_{1} X_{0} X_{3}+a_{0} X_{1} X_{3}\right)+2 \lambda_{3}\left(b_{0} X_{0}+b_{1} X_{1}+b_{2} X_{2}\right) X_{3}=0
\end{gathered}
$$

to be a conic. The quadrics of this web have six common tangent planes. Of the 16 nodes, 10 lie on $V_{6}^{10}$ and 6 are due to contact with $V_{8}^{4}$ by the solid representing the web. Actually these last six nodes all lie on the trope $\lambda_{3}=0$, and all the other tropes contain 4 nodes on $V_{6}^{10}$ and two contact nodes. The quadrics for which $\lambda_{3}=0$ are

1 O. Hesse, Werke, p. 376 = Journal für Math., 49 (185̃5), p. 279. 
inscribed in the cubic developable determined by the six common tangent planes. Conversely it can be shewn that any web with six common tangent planes may be regarded as of the form (1).

Godeaux (loc. cit., §19) starts with a web of quadrics through six points, and proves geometrically that the representative solid of the web meets $V_{8}^{4}$ in a Kummer Surface. He uses the trope which does not meet $V_{6}^{10}$ to represent the twisted cubic through the six points.

\section{§7. The Polar Porism.}

(i) Consider the tangential pencil

$\left(\lambda A_{00}+\mu B_{00}\right) X_{0}^{2}+\left(\lambda A_{11}+\mu B_{11}\right) X_{1}^{2}+\left(\lambda A_{22}+\mu B_{22}\right) X_{2}^{2}+\left(\lambda A_{33}+\mu B_{33}\right) X_{3}^{2}=0$

which we call $\Omega$, and let the line representing it be $l$. Any quadric which forms with $\Omega$ a polar net (i.e. part of the polar system of a class cubic surface) is represented by a point $Q_{c}$ of a plane through $l$ lying on a certain quadric solid cone. The plane $Q_{c} l$ may be regarded as a perfectly general plane representing a polar net and will be denoted by $a$. The 4-secant solid through $l$ we denote by $\Sigma$.

For if a represent a polar net, we may suppose that there is a class cubic surface with respect to which

$\Pi_{1}$ is the plane whose polar quadric, $A$, is $A_{00} X_{0}^{2}+\ldots=0$,

$\Pi_{2}$ is the plane whose polar quadric, $B$, is $B_{00} X_{0}^{2}+\ldots=0$, and $\Pi_{3}$ is the plane whose polar quadric, $C$, is represented by $Q_{c}$. If we write down the conditions for the pole of $\mathrm{I}_{1}$ with respect to $B$ to be the same as that of $\Pi_{2}$ with respect to $A$, and two similar sets, and eliminate the ratios of the coordinates of $\Pi_{1}, \Pi_{2}, \Pi_{3}$, then we find that the coordinates of $Q_{c}$ satisfy a twelfth-order skew-symmetrical determinantal equation reducible to

$$
\begin{gathered}
\left(\lambda y_{01} y_{23}+\mu y_{02} y_{31}+\nu y_{03} y_{12}\right)^{2}=0, \\
\text { where } \lambda \equiv\left(A_{00} B_{11}-A_{11} B_{00}\right)\left(A_{22} B_{33}-A_{33} B_{22}\right) \equiv[01][23], \\
\mu \equiv[02][31], \quad \nu \equiv[03][12] \\
\text { and therefore } \quad \lambda+\mu+\nu \equiv 0 .
\end{gathered}
$$

The quadric (1) is a solid cone $V_{l}$, vertex $\Sigma$. Any point of $V_{3}^{8}$ is on it because of (2). There are $\infty^{1}$ such quadrics with a given vertex, forming a linear pencil whose base is the quartic cone joining $\Sigma$ to $V_{3}^{8}$.

1 See Salmon; Geometry of Three Dimensions (4th ed. 1882), p. 209. 
(ii) There are two systems of [6]'s on $V_{l}$. Those of the first system (3) meet $V_{3}^{8}$ in rational normal sextics through $Q_{00}, Q_{11}, Q_{22}, Q_{33}$, while those of the second, (4), meet $V_{3}^{8}$ in conics and the four isolated points $Q_{00}, Q_{11}, Q_{22}, Q_{33}$. The equations are

and

$$
\left.\begin{array}{l}
b y_{03}-c y_{02}+d \lambda y_{23}=0 \\
c y_{01}-a y_{03}+d \mu y_{31}=0 \\
a y_{02}-b y_{01}+d v y_{12}=0
\end{array}\right\}
$$

$$
\left.\begin{array}{l}
b^{\prime} \nu y_{12}-c^{\prime} \mu y_{13}+d^{\prime} y_{01}=0, \\
c^{\prime} \lambda y_{23}-a^{\prime} \nu y_{12}+d^{\prime} y_{02}=0, \\
a^{\prime} \mu y_{31}-b^{\prime} \lambda y_{32}+d^{\prime} y_{03}=0 .
\end{array}\right\}
$$

There are four exceptional members of the second system (those for which three of $a^{\prime}, b^{\prime}, c^{\prime}, d^{\prime}$, are zero) which meet $V_{3}^{8}$ in a Veronese surface. If we project from $\Sigma$ on to a [5], both systems of [6]'s project into planes meeting the projection of $V_{3}^{8}$ in conics.

If a point pair $P_{x} P_{y}=0$ be represented by $Q_{c}$, and $p$ join the points $P_{x}, P_{y}$, then $p$ is the locus of poles of some plane $\Pi_{3}$ with respect to the quadrics of $\Omega$, since the poles of $\Pi_{1}$ and $\Pi_{2}$ with respect to $C$ are in this case on $p$.

If $p$ is the locus of poles of a plane with respect to quadrics of $\Omega$, the same is true when $p$ is regarded as the join of any other point pair $P_{x}^{\prime} P^{\prime}{ }_{y}$ of $p$, so if $V_{l}$ contains $Q_{x y}$ it also contains the whole $\sigma_{2}$ plane through $Q_{x y}$ and therefore the [6] joining it to $\Sigma$. This [6] is clearly of the second system (4) above.

The lines $p$ form a tetrahedral complex with fundamental tetrahedron $P_{0} P_{1} P_{2} P_{3}$; their Plücker coordinates $\left(l_{1}, m_{1}, n_{1}, \lambda_{1}, \mu_{1}, \nu_{1}\right)$ with respect to this tetrahedron satisfy :-

$$
\frac{l_{1} \lambda_{1}}{\lambda}=\frac{m_{1}}{\mu} \frac{\mu_{1}}{\mu}=\frac{n_{1} \nu_{1}}{\nu}
$$

But the Plücker coordinates of $l$ with respect to the tetrahedron $Q_{00} Q_{11} Q_{22} Q_{33}$ also satisfy (5) and so any line $l^{\prime}$ of the tetrahedral complex in $\Sigma$ thus defined by (5) gives the same quadric (4) as $l$ does. Hence:-

(a) If a quadric represented by $Q_{c}$ forms a polar net with a pencil $\Omega$ represented by $l$, through which the four-secant solid $\Sigma$ passes, then the quadric represented by $Q_{c}$ also forms a polar net with the pencil represented by any other line $l^{\prime}$ which cuts on the faces of $Q_{00} Q_{11} Q_{22} Q_{33} a$ related range. 
(b) The range in which $l$ cuts the faces of $Q_{00} Q_{11} Q_{22} Q_{3_{3}}$ is related to that in which the locus of poles of a plane with respect to quadrics of $\Omega$ cuts the faces of $P_{0} P_{1} P_{2} P_{3}$.

(iii) Any solid lying in a 5-secant [4] of $V_{3}^{8}$ represents the polar system of a class cubic surface. For if the [4] meets $V_{3}^{8}$ in $Q_{00} Q_{11}$ $Q_{22} Q_{33}$ and a point $Q_{44}$, representing the repeated point pair $X_{4}^{2}=0$, and if we arrange that the relation between $X_{0}, X_{1}, X_{2}, X_{3}$ and $X_{4}$ is

$$
X_{0}+X_{1}+X_{2}+X_{3}+X_{4} \equiv 0 \text {, }
$$

then any solid of the [4] represents a web capable of the form

$$
a_{0} X_{0}^{2}+a_{1} X_{1}^{2}+a_{2} X_{2}^{2}+a_{3} X_{3}^{2}+\alpha_{4} X_{4}^{2}=0,
$$

where there is a single linear relation between the $\alpha$ 's, namely

$$
p_{0} \alpha_{0}+p_{1} \alpha_{1}+p_{2} \alpha_{2}+p_{3} \alpha_{3}+p_{4} \alpha_{4}=0 \text {. }
$$

Put $p_{k} \alpha_{k}=\lambda_{k}(k=0,1,2,3,4)$ and the equation of the web can be written

$$
\lambda_{0}\left(\frac{X_{0}^{2}}{a_{0}}\right)+\lambda_{1}\left(\frac{X_{1}^{2}}{a_{1}}\right)+\lambda_{2}\left(\frac{X_{2}^{2}}{a_{2}}\right)+\lambda_{3}\left(\frac{X_{3}^{2}}{a_{3}}\right)+\lambda_{4}\left(\frac{X_{4}^{2}}{a_{4}}\right)=0,
$$

where $\lambda_{0}+\lambda_{1}+\lambda_{2}+\lambda_{3}+\lambda_{4}=0$.

The web $(A)$ is the polar system of the class cubic surface

$$
\frac{X_{0}^{3}}{a_{0}}+\frac{X_{1}^{3}}{a_{1}}+\frac{X_{2}^{3}}{a_{2}}+\frac{X_{3}^{3}}{a_{3}}+\frac{X_{4}^{3}}{a_{4}}=0 .
$$

Any plane in a 5-secant [4] of $V_{3}^{8}$ lies in $\infty^{1}$ sueh solids of that 5-secant [4], and therefore represents part of the polar system of a class cubic surface. It follows that all 5-secant [4]'s of $V_{3}^{8}$ through $l$ lie on $V_{l}$. For suppose one did not. Then we should be able to find in it a plane through $l$ representing part of the polar system of a class cubic surface, which did not lie on $V_{l}$, contrary to what was proved above.

(iv) It remains to consider the question of whether a plane through $l$ known to lie on $V_{l}$ necessarily represents a polar net. To that end we shall shew that in any [6], $S_{6}$, of $V_{l}$ meeting $V_{3}^{8}$ in a sextic [i.e. of system (3)], there is just one 5-secant [4] of $V_{3}^{8}$ through $l$ and an arbitrary point. The sextic $\Gamma_{6}$ in which the [6] with equations (3) meets $V_{3}^{8}$ represents the twisted cubic common to the quadrics

$$
\left.\begin{array}{l}
b x_{0} x_{3}-c x_{0} x_{2}+d \lambda x_{2} x_{3}=0, \\
c x_{0} x_{1}-a x_{0} x_{3}+d \mu x_{3} x_{1}=0 .
\end{array}\right\}
$$


The parametric equations of the sextic are found to be

$$
\begin{aligned}
& y_{00}=\theta^{2}(c \theta-d \lambda)^{2}(c \theta+d \mu)^{2} ; y_{11}=a^{2} \theta^{2}(c \theta-d \lambda)^{2} ; y_{22}=b^{2} \theta^{2}(c \theta+d \mu)^{2} ; \\
& y_{33}=(c \theta-d \lambda)^{2}(c \theta+d \mu)^{2} ; \quad y_{01}=a \theta^{2}(c \theta-d \lambda)^{2}(c \theta+d \mu) ; \\
& y_{02}=b \theta^{2}(c \theta-d \lambda)(c \theta+d \mu)^{2} ; y_{03}=\theta(c \theta-d \lambda)^{2}(c \theta+d \mu)^{2},
\end{aligned}
$$

where $\theta=x_{0} / x_{3}$, and we assume that $d \neq 0$, which we can do without loss of generality, so that $y_{00}, y_{11}, y_{22}, y_{33}, y_{01}, y_{02}, y_{03}$ are independent coordinates for $S_{6}$.

No 5-secant [4] through $l$ can meet $\Sigma$ in a plane unless it contains $\Sigma$ entirely. For otherwise, let $\Sigma_{1}$ be the [5] joining the [4] in question to $\Sigma$. There will be a system of $\infty^{3}$ locus quadrics apolar to all those represented by points of $\Sigma_{1}$, and this system will have nine non-coplanar common points, which is impossible.

Suppose the 5-secant [4] $U$ through $l$ to be given as the intersection of the [5]'s $U Q_{00}$ and $U Q_{33}$, primes of $S_{6}$. Then the equations of $U$ may be written

$$
\left.\begin{array}{r}
{[23] y_{11}+[31] y_{22}+[12] y_{33}+P_{01} y_{01}+P_{02} y_{02}+P_{03} y_{03}=0} \\
{[12] y_{00}+[20] y_{11}+[01] y_{22}+\quad+P^{\prime}{ }_{01} y_{01}+P^{\prime}{ }_{02} y_{02}+P^{\prime}{ }_{03} y_{03}=0,}
\end{array}\right\}
$$

seeing that $U Q_{00}$ meets $\Sigma$ in the plane $l Q_{00}$ whose equations in $S_{6}$ are

$$
[23] y_{11}+[31] y_{22}+[12] y_{33}=y_{01}=y_{02}=y_{03}=0 \text {. }
$$

The parameter of $Q_{00}$ is $\infty$ and that of $Q_{33}$ is 0 . If we substitute the parameters into the equations of $U Q_{00}$ and $U Q_{33}$ and then divide the latter by $\theta$, we get two quintic polynomials in $\theta$ which both give the parameters of the intersections of $U$ and $V_{3}^{8}$, and are therefore proportional. We therefore have the identity

$$
\begin{aligned}
& a^{2}[23] \theta^{2}(c \theta-d \lambda)^{2}+b^{2}[31] \theta^{2}(c \theta+d \mu)^{2}+[12](c \theta-d \lambda)^{2}(c \theta+d \mu)^{2} \\
& +\theta(c \theta-d \lambda)(c \theta+d \mu)\left[a P_{01} \theta(c \theta-d \lambda)+b P_{02} \theta(c \theta+d \mu)+P_{03}(c \theta-d \lambda)(c \theta+d \mu)\right] \\
& \equiv K\left\{[12] \theta(c \theta+d \lambda)^{2}(c \theta-d \mu)^{2}+a^{2}[20](c \theta-d \lambda)^{2}+b^{2}[01] \theta(c \theta+d \mu)^{2}\right. \\
& \left.\quad+(c \theta-d \lambda)(c \theta+d \mu)\left[a P^{\prime}{ }_{01} \theta(c \theta-d \lambda)+b P_{.02}^{\prime} \theta(c \theta+d \mu)+P_{03}^{\prime}(c \theta-d \lambda)(c \theta+d \mu)\right]\right\} .
\end{aligned}
$$

Putting $\theta=0$ in both sides gives

$$
K P_{03}^{\prime} \equiv[12] \text {. }
$$

Putting $c \theta=d[0]][23]$ or $c \theta=-d[02][31]$ gives

$$
K=d[23][31] / c \text {. }
$$


If we now divide the identity by $\theta(c \theta-d \lambda)(c \theta+d \mu)$ we reduce it to $a P_{01} \theta(c \theta-d \lambda)+b P_{02} \theta(c \theta+d \mu)+P_{03}(c \theta-d \lambda)(c \theta+d \mu)$

$-(d / c)[23][31]\left\{(c \theta-d \lambda)(c \theta+d \mu)+a P^{\prime}{ }_{01}(c \theta-d \lambda)+b P^{\prime}{ }_{02}(c \theta+d \mu)\right\}$ $+(1 / c)\left\{a^{2}[23](c \theta-d \lambda)+b^{2}[31](c \theta+d \mu)\right\} \equiv 0$.

As this is quadratic in $\theta$, it will be sufficient to express the fact that it has three roots $0, \frac{d \lambda}{c},-\frac{d \mu}{c}$. Hence we obtain by putting $\theta=0$,

$-P_{03} d[01][02]+\left(d^{2} / c\right)[01][02][03][31]+(a d / c)[01][23] P^{\prime}{ }_{01}$

$-(b d / c)[02][31] P_{02}^{\prime}-\left(a^{2} / c\right)[01][23]^{2}+\left(b^{2} / c\right)[02][31]^{2}=0$.

By putting $c \theta=d \lambda$ we obtain

$$
d[01][23] P_{02}-d[23][31] P_{02}^{\prime}+b[31]=0,
$$

and by putting $c \theta=-d \mu$ we obtain

$$
d[02][31] P_{01}+d[23][31] P_{01}^{\prime}-a[23]=0 .
$$

(1) (2)(3)(4) are 4 linear equations between the non-homogeneous coefficients $P_{01}, P_{02}, P_{03}, P_{01}^{\prime}, P_{02}^{\prime}, P_{03}^{\prime}$. The conditions for the [4] $U$ to go through an arbitrary point of $S_{6}$ not on $l$ would give 2 further linear relations, and therefore there is in general a unique solution, i.e. through a plane a containing a line $l$ and on $V_{l}$ there is one 5-secant [4] of $V_{3}^{8}$ in each space $S_{6}$ containing a.

Since there are $\infty^{1}$ spaces $S_{6}$ on $V_{l}$ through $\alpha$, there are therefore $\infty^{1} 5$-secant [4]'s of $V_{3}^{8}$ through a [N.B. 2 spaces $S_{6}$ do not intersect in a 5-secant [4] of $V_{3}^{8}$ ].

We have now proved:

(1) A necessary condition for a plane a to represent a polar net is that it lie on the quadric $V_{l}$ defined as above for any line $l$ in a. Hence the general plane of [9] does not represent a polar net.

(2) If a plane a lies on $V_{l}$ and $l$ is in a, then in each space $S_{6}$ on $V_{l}$ through a there is one 5-secant [4] of $V_{3}^{8}$ through a. Hence there are $\infty^{1} 5$-secant [4]'s of $V_{3}^{8}$ through a altogether. In each of the 5-secant [4]'s there are $\infty^{1}$ solids through $a$, and therefore [ 7 (iii)] we can find $\infty^{2}$ cubic surfaces to whose polar systems the net represented by a belongs. We have thus found a geometrical reason in [9] for the fact that the condition for a net to be polar is poristic.

( $\nabla)$ It is possible also to shew in this way that self-conjugate pentagrams, for a net of quadrics which is polar, form a $g_{5}^{1}$ on a twisted 
cubic. ${ }^{1}$ For we can prove that the [6], $T$, which joins two 5-secant [4]'s through a contains them all, and that it cannot degenerate into a [5]. $T$ meets $V_{3}^{8}$ in a curve which must be a sextic, also $T$ lies in the prime $P$ which touches $V_{l}$ all along $a$. All spaces $S_{6}$ through a lie in $P$ and any two meet in $[S a]$, which is not a 5-secant [4] of $V_{3}^{8}$. Hence the sextics in which these spaces $S_{6}$ meet $V_{3}^{8}$ represent twisted cubics of the same system on the locus quadric $Q$ representing $P$. It can be shewn that $T$, which lies in $P$, meets $V_{3}^{8}$ in a sextic representing a twisted cubic of the opposite system on $Q$, which therefore meets every one of the cubics represented by intersections of spaces $S_{6}$ with $V_{3}^{8}$ in 5 points. The [4]'s joining such sets of 5 points generate the quadric plane cone in which $T$ meets $V_{l}$. The fact that sets of points representing self-conjugate pentagrams lie on [4]'s which generate a quadric plane cone proves that they form a $g_{5}^{1}$ on the twisted cubic represented by the intersection of $T$ and $V_{3}^{8}$.

Conversely, if we consider the $g_{5}^{1}$ on the twisted cubic which is the locus of $\left(\theta^{3}, \theta^{2}, \theta, 1\right)$ given by $\left(a_{0}+\lambda a_{0}^{\prime}\right) \theta^{5}+\left(a_{1}+\lambda a_{1}^{\prime}\right) \theta^{4}+\left(a_{2}+\lambda a_{2}^{\prime}\right) \theta^{3}+\left(a_{3}+\lambda a_{3}^{\prime}\right) \theta^{2}+\left(a_{4}+\lambda a_{4}^{\prime}\right) \theta+\left(a_{5}+\lambda a_{5}^{\prime}\right)=0$, we find that the net of quadrics having the sets of this $g_{5}^{1}$ for selfconjugate pentagrams is represented by the plane

$$
\left.\begin{array}{l}
a_{0} y_{0}+a_{1} y_{1}+a_{2} y_{2}+a_{3} y_{3}+a_{4} y_{4}+a_{5} y_{5}=0, \\
a_{0} y_{1}+a_{1} y_{2}+a_{2} y_{3}+a_{3} y_{4}+a_{4} y_{5}+a_{5} y_{6}=0, \\
a_{0}^{\prime} y_{0}+a_{1}^{\prime} y_{1}+a_{2}^{\prime} y_{2}+a_{3}^{\prime} y_{3}+a_{4}^{\prime} y_{4}+a^{\prime} y_{5}=0, \\
a_{0}^{\prime} y_{1}+a_{1}^{\prime} y_{2}+a_{2}^{\prime} y_{3}+a_{3}^{\prime} y_{4}+a^{\prime}{ }_{4} y_{5}+a_{5}^{\prime} y_{6}=0,
\end{array}\right\}
$$

where $y_{0}, \ldots, y_{6}$ are coordinates in the space of the sextic representing the locus of $\left(\theta^{3}, \theta^{2}, \theta, 1\right)$ so chosen that the parametric equations to this sextic are

$$
y_{0}: y_{1}: y_{2}: y_{3}: y_{4}: y_{5}: y_{6}=\theta^{6}: \theta^{5}: \theta^{4}: \theta^{3}: \theta^{2}: \theta: 1
$$

1 See Edge "A special net of quadrics," Proc. Edin. Math. Soc. (2) 4 (1936) 185. 